\title{
Economic Evaluation of Linezolid versus Vancomycin in Mechanical Ventilation-Associated Nosocomial Pneumonia Caused by Methicillin-Resistant Staphylococcus aureus
}

\author{
Tazio Vanni \\ Faculty of Medicine of Ribeirão Preto, University of São Paulo, Brazil
}

Economic analyses of treatment options are particularly important to assist treatment decisions in a resource-limited country such as Brazil. Recently, Machado et al.published in this journal a cost-effectiveness analysis (CEA), comparing linezolid versus vancomycin for the treatment of mechanical ventilation-associated nosocomial pneumonia caused by methicillin-resistant Staphylococcus aureus (MRSA) [1]. From a public hospital perspective, they concluded that, despite the higher price per unit, linezolid was more cost-effective than vancomycin. Even though, the results appear to be promising, there are a few limitations of this study that should be considered when interpreting its results.

The decision tree used in this study lacks in balance, since it did not incorporate the down side (e.g., risk of side effects) of the treatment options in the comparison [2]. Even if Brazilian data on the occurrence of adverse effects of these two drugs are not available, data derived from international publications could have been used in the analysis [3,4]. Both linezolid and vancomycin have been associated with myelosuppression and other adverse effects, which can increase the total patient-treatment cost [5].

Different from other published CEA regarding this issue, the Machado et al. study did not present a sensitivity analysis, which could jeopardize the robustness of its results [6,7]. Sensitivity analysis is the standard way of dealing with uncertainties in the CEA literature. In a sensitivity analysis, critical components in the calculation are changed by a meaningful amount or varied from worst case to best case, and the cost-effectiveness ratio is recalculated [8].

As reported in the study by Machado et al., when estimating direct total cost, the drug administration cost, the nurse monitoring cost, and other similar medical expenses normally should be incorporated into the economic analysis [8]. In this case, these costs would not have great impact on the results, but it is always useful to have accurate data, which could be used as a reference for future publications.

In future studies, efforts should be made to estimate long-term treatment costs and benefits (i.e. outcomes, such as qualityadjusted life years gained). The possibility of sequential intravenous-to-oral therapy of linezolid, facilitating early discharge from hospital, should also be explored in future economic evaluations. The emergence of resistance is another variable to be incorporated into decision-analysis models. In addition, as much as possible, research groups should avoid any conflict of interests that could affect the credibility of their studies, and they should always report their source of funding.

\section{References}

1. Machado A.R., Arns C. de C., Follador W., Guerra A. Cost-effectiveness of linezolid versus vancomycin in mechanical ventilationassociated nosocomial pneumonia caused by methicillin-resistant Staphylococcus aureus. Braz J Infect Dis 2005;9(3):191-200.

2. Detsky A.S., Naglie G., Krahn M.D., et al. Primer on medical decision analysis: part 2 - Building a tree. Med Decis Making 1997;17:12635.

3. Rubinstein E., Cammarata S., Oliphant T., Wunderink R. Linezolid (PNU-100766) versus vancomycin in the treatment of hospitalized patients with nosocomial pneumonia: a randomized, double-blind, multicenter study. Clin Infect Dis 2001;32:402-12.

4. Rubinstein E., Isturiz R., Standiford H.C., et al. Worldwide assessment of linezolid's clinical safety and tolerability: comparatorcontrolled phase III studies. Antimicrob Agents Chemother 2003;47(6):1824-31.

5. Plosker G. L., Figgitt D.P. Linezolid: a pharmacoeconomic review of its use in serious Gram-positive infections. Pharmacoeconomics 2005;23(9):945-64.

6. Shorr A.F., Susla G.M., Kollef M.H. Linezolid for the treatment of ventilator-associated pneumonia: a cost-effective alternative to vancomycin. Crit Care Med 2004;32(1):137-43.

7. Grau S., Alvarez-Lerma F., del Castillo A., et al. Cost-effectiveness analysis of the treatment of ventilator-associated pneumonia with linezolid or vancomycin in Spain. J Chemother 2005:17(2);203-11.

8. Gold M.R., Siegel J.E., Russell L.B., Weinstein M.C. Cost-effectiveness in health and medicine. New York, US: Oxford University Press, 1996. 